\title{
Safety and Efficacy of Tirofiban in Acute Ischemic Stroke Patients Receiving Endovascular Treatment: A Meta-Analysis
}

\author{
Zhiyong Fu Chuanli Xu Xin Liu Zhengze Wang Lianbo Gao \\ Department of Neurology, The Fourth Affiliated Hospital of China Medical University, Shenyang, China
}

\section{Keywords}

Ischemic stroke · Endovascular treatment · Tirofiban

\begin{abstract}
Objectives: Tirofiban is widely used in clinical practice for acute ischemic stroke (AIS). However, whether tirofiban increases the bleeding risk or improves the outcome of AIS patients with endovascular treatment (ET) is unknown. The aim of this meta-analysis is to evaluate the safety and efficacy of tirofiban compared with those without tirofiban in AIS patients receiving ET. Methods: Systematic literature search was done in PubMed and EMBASE databases without language or time limitation. Safety outcomes were symptomatic intracranial hemorrhage $(\mathrm{sICH})$ and mortality. Efficacy outcomes were recanalization rate and favorable functional outcome. Review Manager 5.3 and Stata Software Package 15.0 were used to perform the meta-analysis. $\boldsymbol{R e}$ sults: Eleven studies with a total of 2,028 patients were included. A total of 704 (34.7\%) patients were administrated tirofiban combined with ET. Meta-analysis suggested that tirofiban did not increase the risk of sICH (odds ratio (OR) 1.08; 95\% confidence interval $(\mathrm{Cl}) 0.81-1.46 ; p=0.59)$ but significantly decreased mortality (OR $0.68 ; 95 \%$ Cl 0.52-0.89; $p=0.005)$. There was no association between tirofiban and
\end{abstract}

\section{KARGER}

() 2020 S. Karger AG, Basel recanalization rate (OR 1.26; $95 \% \mathrm{Cl} 0.86-1.82 ; p=0.23$ ) or favorable functional outcome (OR 1.21 ; $95 \% \mathrm{Cl} 0.88-1.68$; $p=0.24)$. Subgroup analyses indicated that preoperative tirofiban significantly increase recanalization rate (OR 3.89; $95 \% \mathrm{Cl} 1.70-8.93 ; p=0.001)$ and improve favorable functional outcome (OR 2.30; 95\% Cl 1.15-4.60; $p=0.02$ ). Conclusions: Tirofiban is safe in AIS patients with ET and can significantly reduce mortality; preoperative tirofiban may be effective, but further studies are needed to confirm the efficacy.

(c) 2020 S. Karger AG, Basel

\section{Introduction}

Endovascular treatment (ET) has been proved to be an effective therapy to improve functional outcomes in selected patients with acute ischemic stroke (AIS) [1-6]. However, platelet activation caused by endothelial injuries may lead to thromboembolic complications and give rise to early reocclusion during the operative procedure, $[7,8]$. Tirofiban, a highly selective glycoprotein (GP) IIb/ IIIa receptor antagonist, which potently inhibits the final pathway of platelet activation, has already been widely used in recent clinical practice to prevent early thrombosis in ET for AIS [9]. However, as an off-label usage, 


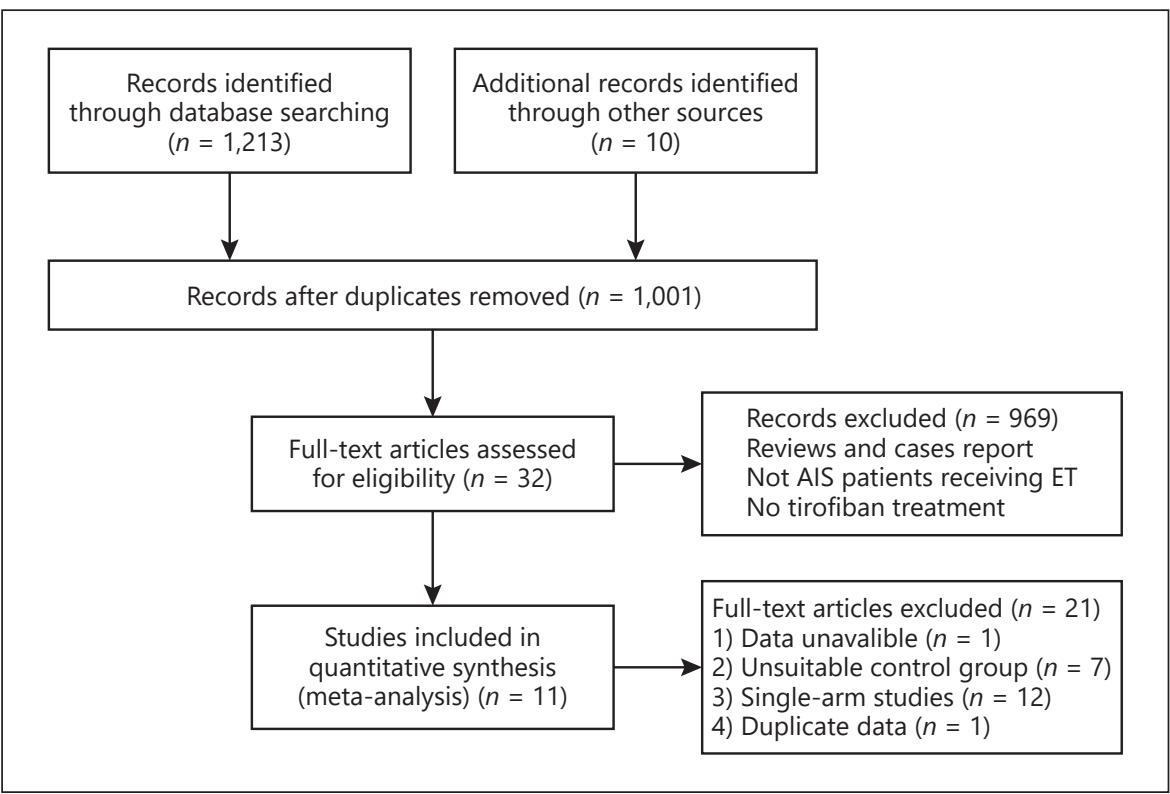

Fig. 1. Flow chart of the screening process.

whether tirofiban increases the bleeding risk or improves the outcome of ET in AIS is unknown. There were several studies that evaluated the feasibility of tirofiban in AIS patients receiving ET, and the results were conflicting [10-20]. Thus, we aimed to perform a meta-analysis to evaluate the safety and efficacy of tirofiban in combination with ET for AIS patients.

\section{Methods}

\section{Search Strategy}

An electronic database search was conducted by using subject term (stroke, tirofiban) combined with its free term in the following databases: MEDLINE via PubMed and EMBASE (without language or time limitation). The systematic literature search was ultimately performed in October 2019. Detailed electronic search strategy for PubMed was exemplified in suppl. Table 2 (for all online suppl. material, see www.karger.com/doi/10.1159/000509054). The database searches were accompanied by hand searches of the reference list of included articles, as well as contacting authors for all included and potentially included studies. The gray literature and ongoing studies were searched in the following databases: OpenGrey, WHO International Clinical Trials Registry Platform, and ClinicalTrials.Gov. Two reviewers who blind to each other independently screened the titles and abstracts to access their eligibility. Full texts of potentially eligible citations were retrieved for detailed examination.

\section{Study Selection}

Studies were screened and extracted by 2 reviewers (ZhiYong $\mathrm{Fu}$ and ChuanLi $\mathrm{Xu}$ ) independently according to inclusion and exclusion criteria, and then cross-checked. Disagreements regarding extracted data were resolved by discussion among the authors.
If necessary, a third author was required to assess the remaining disagreements. Inclusion criteria: (1) observational studies or randomized controlled trials (RCTs); (2) studies of tirofiban on AIS patients receiving ET compared to patients without tirofiban. Exclusion criteria: (1) studies of single-arm trial or cases report; (2) reviews and studies from which abstracts or data could not be extracted; (3) duplicate reporting came from a same trail.

\section{Data Extraction and Quality Assessment}

Data extraction: (1) baseline of the included studies: age, male, hypertention, diabetes mellitus, atrial fibrillation, coronary heart disease, and previous stroke; (2) studies' characteristics: author, year, country, trial design, sample size, heparin usage, administration route of tirofiban, safety outcomes, and efficacy outcomes. We contacted corresponding authors via e-mail to request further information when necessary. As all included studies were observational studies, the risk of bias was assessed by the Newcastle-Ottawa Scale (NOS). High-quality study was defined as having an NOS ranking of 7-9 [21].

\section{Outcome Measure}

There were 2 primary safety outcome end points: symptomatic intracranial hemorrhage ( $\mathrm{sICH}$ ) and mortality. sICH was defined according to the definition of the European Cooperative Acute Stroke Study III [22]. Mortality was 3-month follow-up mortality. There were also 2 primary efficacy outcome end points: recanalization rate and favorable functional outcome. Recanalization was defined as a thrombolysis in cerebral infarction score of 2b-3 [23]. Favorable functional outcome was defined as a modified Rankin Scale (mRS) of 0-2 at 3-month follow up [24].

\section{Statistical Analysis}

Data were entered and analyzed by using the Cochrane Collaboration Review Manager software (version 5.3) and Stata Software Package (version 15.0). The data of end points were analyzed 
Table 1. Characteristics of included studies

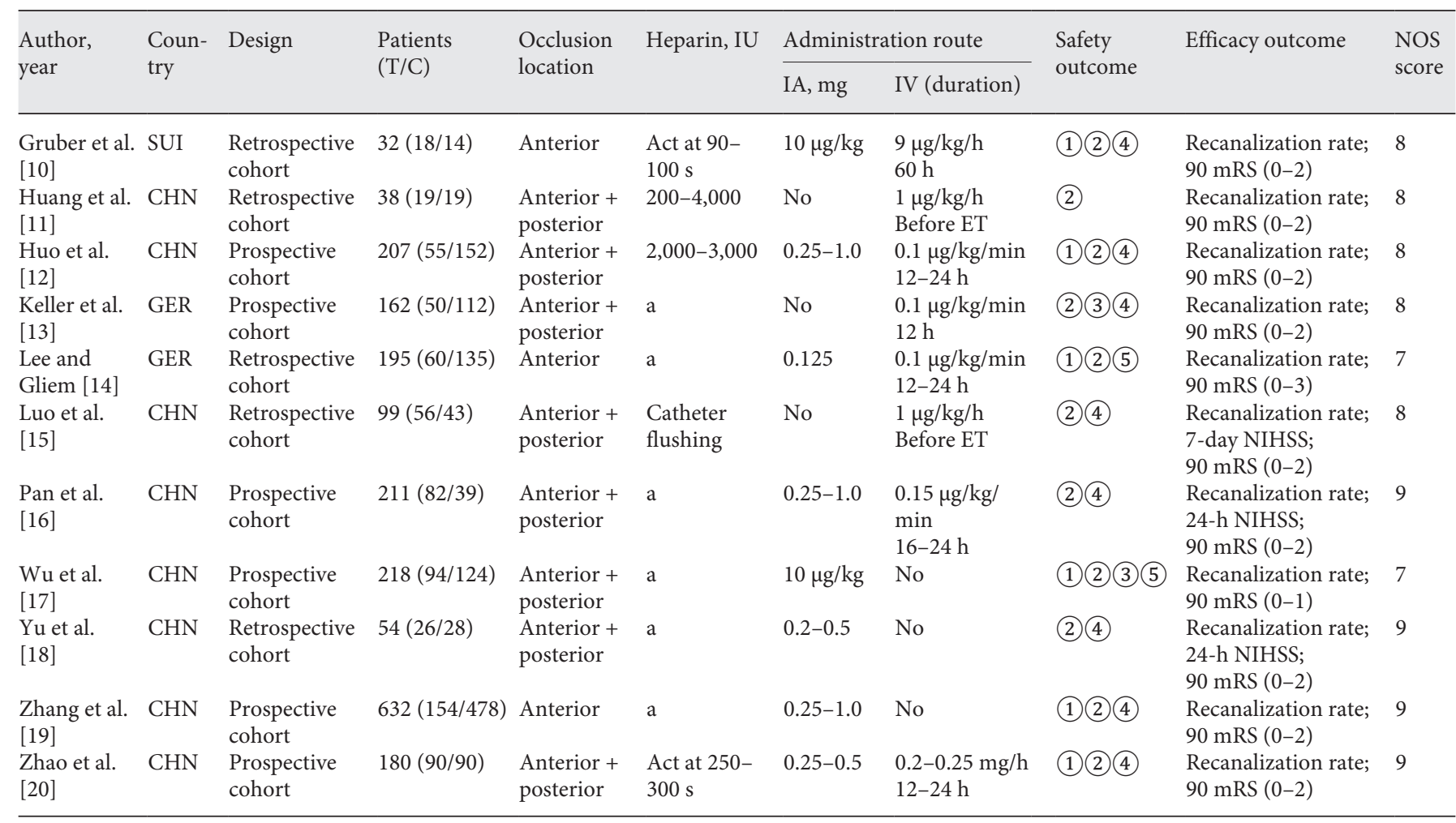

High-quality study was defined as having an NOS ranking of 7-9. T, tirofiban group; C, control group; IA, intra-arterial; IV, intravenous; ET, endovascular treatment; ACT, activated clotting time; a, detailed information was not reported; (1), any ICH; (2), sICH; (3), fatal ICH; (4), 3-month mortality; (5), hospital death; NOS, Newcastle-Ottawa Scale; mRS, modified Rankin Scale.

separately by indications ( $\mathrm{sICH}$, mortality, recanalization rate, and favorable functional outcome). Odds ratios (ORs) and 95\% confidence intervals (CIs) were calculated by using the Mantel-Haenszel method with random-effects model if $I^{2}>50 \%$ or $p \leq 0.10$, with fixed-effects model if $I^{2}<50 \%$ and $p>0.10$. In case of heterogeneity $\left(I^{2}>50 \%\right.$ or $\left.p \leq 0.10\right)$, we attempted to identify and explain it by using subgroup analysis [25]. Sensitivity analysis was performed separately by excluding the study with the largest sample size [19], excluding the study with the smallest sample size [10], then excluding the only one study indicating significant difference in $\mathrm{sICH}$ [17], and excluding one study indicating significant difference in recanalization rate between the tirofiban group and without the tirofiban group [14]. Funnel plots and Begg's linear regression test were used to evaluate publication bias. $p$ values were considered statistically significant for $p<0.05$.

\section{Results}

\section{Search Results}

The systematic review and meta-analysis were prepared following the PRISMA. We retrieved 1,213 articles that were potentially pertinent. After removing duplicates and reviewing titles and abstracts to exclude irrelevant studies, case reports, and reviews, 11 cohort trials [10-20] fulfilled all the inclusion criteria and included 2,028 patients (Fig. 1).

\section{Study Characteristics}

Baseline of the included studies (age, male, hypertention, diabetes mellitus, atrial fibrillation, coronary heart disease, and previous stroke) is summarized in online suppl. Table 1. The enrolled studies' characteristics and analyzed NOS score are presented in Table 1. According to the administration route of tirofiban [9], the 11 studies can be divided into 2 subgroups: (1) rescue tirofiban: 9 studies used tirofiban during or after endovascular procedures; (2) preoperative tirofiban: $2[11,15]$ studies used tirofiban before endovascular procedures.

\section{Quantitative Data Synthesis \\ Safety Outcomes \\ sICH}

The overall sICH occurrence was 232 (11.4\%), of which $81(11.5 \%)$ pertain to the tirofiban group and 151 


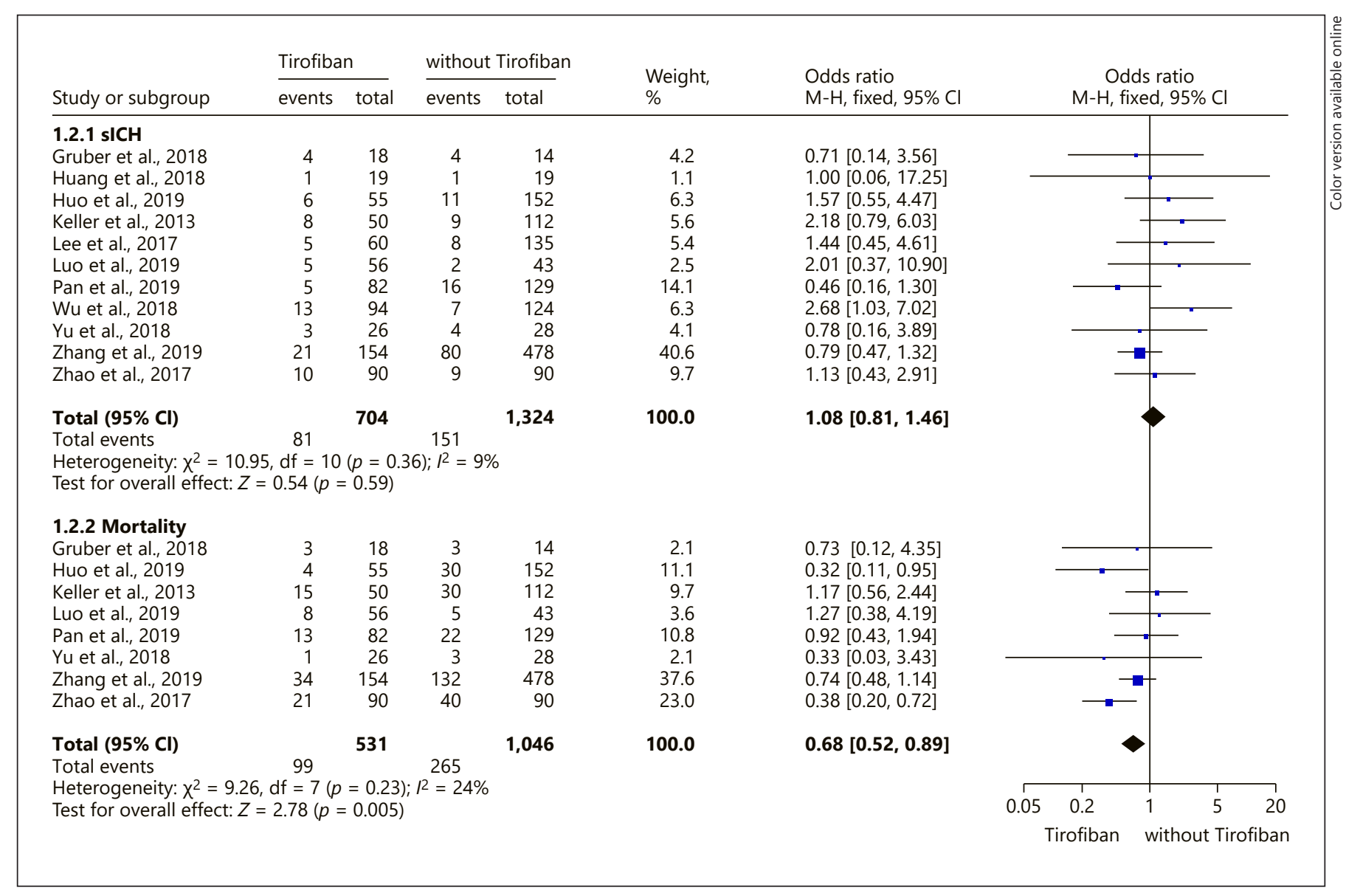

Fig. 2. Forest plot of safety outcomes in patients with and without tirofiban.Safety outcomes include sICH and mortality. M-H fixed: Mantel-Haenszel method with fixed-effects model.

(11.4\%) pertain to the without tirofiban group. Only one study [17] demonstrated a significantly increased risk of sICH in the tirofiban group compared with the without tirofiban $(p=0.027)$. When estimating the relationship between tirofiban and $\mathrm{sICH}$, pooled analysis showed that tirofiban did not increase the risk of sICH in patients with ET (OR 1.08; 95\% CI 0.81-1.46; $p=0.59$ ). The heterogeneity $\left(p=0.36 ; I^{2}=9 \%\right)$ between these studies was low (Fig. 2).

\section{Mortality}

Eight studies investigated the 3-month follow-up mortality after ET. The overall mortality occurrence was 364 (23.1\%), of which 99 (18.6\%) relate to the tirofiban group and $265(23.3 \%)$ relate to the without tirofiban group. There were 2 studies $[12,20]$ that indicated significant relationship between tirofiban and mortality. Pooled analysis demonstrated that tirofiban can significantly re- duce mortality (OR 0.68 ; 95\% CI $0.52-0.89 ; p=0.005$ ). The heterogeneity $\left(p=0.23 ; I^{2}=24 \%\right)$ between these studies was low (Fig. 2).

\section{Efficacy Outcomes}

\section{Recanalization Rate}

A total of 11 studies reported early recanalization rate after ET. The overall recanalization occurrence was 1,670 $(82.3 \%)$, of which $591(83.9 \%)$ connected with the tirofiban group and $1,079(81.5 \%)$ pertain to the without tirofiban group. One study [14] showed a significantly increased recanalization rate in the tirofiban group compared with the without tirofiban group $(p=0.026)$. There was also 1 study [11] that illuminated preoperative tirofiban can increase the recanalization rate $(p=0.004)$. Another 7 studies showed no significant relationship between tirofiban and recanalization. Pooled analysis found tirofiban was not associated with early recanalization in 


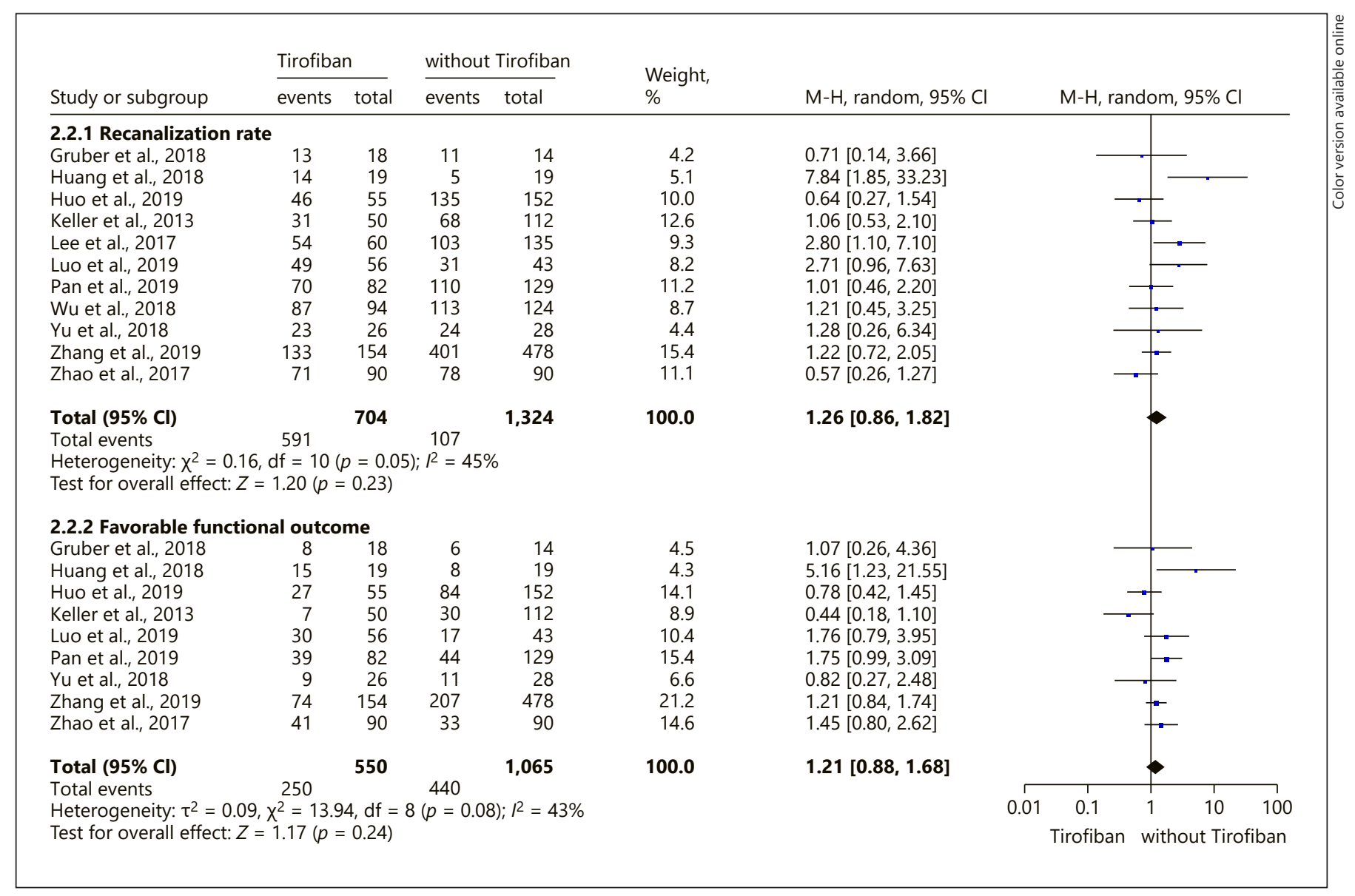

Fig. 3. Forest plot of efficacy outcomes in patients with and without tirofiban.Efficacy outcomes include recanalization and favorable functional outcome. M-H random: Mantel-Haenszel method with random-effects model.

patients with ET (OR 1.26; 95\% CI 0.86-1.82; $p=0.23$ ). The $I^{2}$ value was $45 \%$, while $p$ value $<0.1$ (Fig. 3 ).

Favorable Functional Outcome

Nine studies reported favorable functional outcome defined as an mRS of 0-2 at 3-month follow up, while one study [17] defined as an mRS of $0-1$ and also one study [14] defined as an $\mathrm{mRS}$ of $0-3$. The overall favorable functional outcome (mRS 0-2) occurrence following ET was 690 (42.7\%), of which 250 (45.5\%) associated with the tirofiban group and $440(41.3 \%)$ pertain to the without tirofiban group. There was only 1 study [11] that indicated preoperative tirofiban was associated with favorable functional outcome in patients who received ET ( $p=$ 0.02 ). Pooled analysis indicated that tirofiban was not associated with favorable functional outcome (OR 1.21; $95 \%$ CI $0.88-1.68 ; p=0.24)$. The $I^{2}$ value was $43 \%$, while $p$ value $<0.1$ (Fig. 3 ).
Subgroup Analysis of Efficacy Outcome

In consideration of the heterogeneity (recanalization rate: $I^{2}=45 \%, p<0.1$; favorable functional outcome: $I^{2}=$ $43 \%, p<0.1$ ), we performed subgroup analysis. 11 studies were divided into 2 subgroups according to the administration route of tirofiban: preoperative tirofiban subgroup ( 2 studies) and rescue tirofiban subgroup (9 studies). Pooled analysis suggested that preoperative tirofiban can significantly increase the recanalization rate $(\mathrm{OR}$ 3.89; 95\% CI 1.70-8.93; $p=0.001 ; I^{2}=27 \%$; online suppl. Fig. 1) and can also significantly improve favorable functional outcome (OR 2.3; 95\% CI 1.15-4.60; $p=0.02 ; I^{2}=$ 39\%; online suppl. Fig. 2).

\section{Sensitivity Analysis}

Following the method described in the Statistical Analysis section, the influence analysis consistently omitted one study at a time. None of the studies influenced the 
Table 2. Sensitivity analysis for sICH and mortality

\begin{tabular}{|c|c|c|c|c|c|c|}
\hline & \multicolumn{3}{|l|}{ sICH } & \multicolumn{3}{|l|}{ Mortality } \\
\hline & OR (95\%) & $I^{2}, \%$ & $p$ value & OR (95\%) & $I^{2}, \%$ & $p$ value \\
\hline Zhang et al. [19] & $1.29(0.89-1.86)$ & 0 & 0.18 & $0.65(0.46-0.91)$ & 34 & 0.01 \\
\hline Gruber et al. [10] & $1.10(0.81-1.49)$ & 16 & 0.53 & $0.68(0.52-0.90)$ & 35 & 0.006 \\
\hline Wu et al. [17] & $0.98(0.71-1.34)$ & 0 & 0.89 & $0.68(0.52-0.89)$ & 24 & 0.005 \\
\hline Lee and Gliem [14] & $1.06(0.78-1.45)$ & 16 & 0.69 & $0.68(0.52-0.89)$ & 24 & 0.005 \\
\hline
\end{tabular}

Zhang et al. [19], excluding the study with largest sample size; Gruber et al. [10], excluding the study with smallest sample size; Wu et al. [17], excluding the only one study indicated significant difference in sICH; Lee and Gliem [14], excluding one study indicated significant difference in recanalization rate. OR, odds ratio; $I^{2}$, $I^{2}$ value; sICH, symptomatic intracerebral hemorrhage.

Table 3. Sensitivity analysis for recanalization rate and favorable outcome

\begin{tabular}{|c|c|c|c|c|c|c|}
\hline & \multicolumn{3}{|c|}{ Recanalization rate } & \multicolumn{3}{|c|}{ Favorable functional outcome } \\
\hline & OR (95\%) & $I^{2}, \%$ & $p$ value & OR (95\%) & $I^{2}, \%$ & $p$ value \\
\hline Zhang et al. [19] & $1.28(0.82-2.00)$ & 50 & 0.28 & $1.21(0.80-1.84)$ & 50 & 0.36 \\
\hline Gruber et al. [10] & $1.29(0.87-1.91)$ & 49 & 0.20 & $1.22(0.86-1.73)$ & 50 & 0.26 \\
\hline Wu et al. [17] & $1.27(0.84-1.92)$ & 50 & 0.26 & $1.21(0.88-1.68)$ & 43 & 0.24 \\
\hline Lee and Gliem [14] & $1.15(0.79-1.66)$ & 38 & 0.46 & $1.21(0.88-1.68)$ & 43 & 0.24 \\
\hline
\end{tabular}

Favorable functional outcome, defined as a modified Rankin Scale of 0-2 at 3-month follow-up; Zhang et al. [19], excluding the study with largest sample size; Gruber et al. [10], excluding the study with smallest sample size; Wu et al. [17], excluding the only one study indicated significant difference in sICH; Lee and Gliem [14], excluding one study indicated significant difference in recanalization ate. OR, odds ratio; $I^{2}, I^{2}$ value.

results for any end points such that the results would have changed significantly. The results of sensitivity analysis are shown in Tables 2 and 3 and online suppl. Figure 3.

\section{Publication Bias}

The funnel plot was performed to assess the publication bias (online suppl. Figs. 1-7). Visual inspection of the funnel plot and Begg's test indicated no evidence of publication bias.

\section{Discussion}

In this meta-analysis, we found that tirofiban did not increase the risk of sICH but can significantly reduce mortality in AIS patients undergoing ET, and it did not improve the recanalization rate and favorable functional outcome. However, subgroup analyses indicated that preoperative tirofiban may be effective as it was associated with an increase in recanalization rate and favorable functional outcome.
Tirofiban has been extensively used as monotherapy in progressive stroke, combining intravenous thrombolysis and ET in both preclinical and clinical studies. Promising data from the SaTIS [26] and SETIS [27] trials showed that monotherapy of tirofiban was safe and had potential efficacy in AIS patients. However, whether tirofiban is safe or effective in AIS patients treated with ET is unclear. In this meta-analysis, we found that the mortality at 3 months was significantly lower in patients treated with tirofiban than in the control subjects. We speculate that this may be based on positive effects on existing comorbidity such as cardiovascular disease $[26,28]$. Although we cannot conclude that tirofiban can improve artery recanalization or favorable functional outcomes, it is worth noting that preoperative tirofiban may be effective. The heterogeneity between the included studies may be attributed to several reasons. First, differences in screening criteria for patients suitable to ET: (1) Changes of screening criteria in guidelines: In 2015, the clinical guideline of ET for AIS updated after the publication of 5 
stent retriever trials which confirmed the effect of ET in selected patients with AIS [1-6], whereas Kellert et al. [13] conducted the study before 2015 and concluded that tirofiban was associated with increased risk of fatal ICH and poor outcome. However, the results of Zhao et al. [20] and Pan et al. [16], conducted after 2015, were quite the contrary. (2) Discretion of interventionists: the use of tirofiban was at the discretion of interventionists who may be prone to use tirofiban in subjects with heavier atherosclerotic burden and high possibility of reocclusion after the occluded arteries were partially recanaliazed or ultimately achieved good reperfusion. It may explain why rescue tirofiban, indeed effective, cannot be demonstrated to be able to improve recanalization rate and favorable functional outcomes. Second, update of technical materials: all subjects in Zhao et al. [20] and Pan et al. [16] were treated with second-generation stent retriever device using improved thrombectomy technique, whereas almost $20 \%$ of subjects of Kellert et al. [13] used others (e.g., balloon expansion technique, merci retriever, and stent implantation) which may have led to lower recanalization rate and poor outcome [29]. Third, the dose of tirofiban (Zhang et al. [19] and Yu et al. [18]) indicated that a low dose of tirofiban with an intra-arterial bolus followed by continuous intravenous administration may lower the incidence of sICH. Zhao et al. [20] concluded that a lowdose tirofiban can improve long-term functional outcome. More importantly, the administration route of tirofiban (Huang et al. [11] and Luo et al. [15]) showed that preoperative tirofiban may be effective as it was associated with an increase in recanalization rate and favorable functional outcome. The results were also confirmed by other researchers. Mangiafco et al. [30] and Ihn et al. [31] reported that proactive administration of intravenous tirofiban plus local intra-arterial urokinase and/or MT with AIS attributable to large cerebral artery occlusion can potentially improve recanalization at $24 \mathrm{~h}$ after operation and favorable outcomes at 3 months, while not increasing the risk of sICH. Histopathological analysis found that all the thrombi contained different amounts of platelets and fibrinogen, which may be the key reason why the preoperative application of tirofiban can improve recanalization rate by preventing platelet aggregation and vascular reocclusion before, during, and after ET procedures $[15,32,33]$. Given the pharmacological effects of GP IIb/IIIa inhibitors, tirofiban can prevent the formation of microthrombosis and microembolism, which can deteriorate postischemic flow and infarct progression, but tirofiban have no thrombolytic properties, so tirofiban cannot dissolve microthrombosis and micro- embolism [34, 35]. Therefore, we speculate that tirofiban, especially preoperative tirofiban, may improve the functional outcome by improving the reperfusion status of microvascular. As to patients complicated with certain underlying pathogenesis, such as intracranial atherosclerosis, proactive use of tirofiban seems reasonable but awaits further confirmation by RCTs.

Our increasingly improved understanding of the role of tirofiban in the development of AIS has been providing potential alternative perspectives or adjuvants to current treatment. Reductions in mortality and adverse functional outcomes derived from tirofiban can be achieved when neuro-interventionists and neurologists work collaboratively and efficiently. But further studies are needed to determine the optimal patient selection criteria, dose of tirofiban, and the method of tirofiban to establish a standard treatment protocol.

This meta-analysis has several limitations. First, there was no RCT evidence as the meta-analysis only included observational cohort studies. Furthermore, administration of tirofiban has been mainly concentrated in Asian countries nowadays, due to the lower costs compared with other kinds of GP IIb/IIIa such as abciximab, eptifibatide, which were used more commonly in developed counties. Thus, the current study may be more valuable in Asian countries. In addition, the dose and duration of tirofiban used in the 11 studies were pragmatic. And there existed a difference in heparin usage during endovascular procedures. The potential bias may lead to heterogeneity between studies and exert an effect on outcome measures. Finally, there are only 2 studies in the pre-operative tirofiban subgroup, and these results of the current studies should be explained with caution.

\section{Conclusion}

Tirofiban is safe in AIS patients with ET and can significantly reduce mortality. Although our meta-analysis suggested that tirofiban did not improve the recanalization rate and favorable functional outcome, subgroup analyses indicated that preoperative tirofiban may be effective, and further studies are needed to confirm the efficacy.

\section{Statement of Ethics}

Ethical approval was not required for this type of study. 


\section{Conflict of Interest Statement}

The authors have no conflicts of interest to declare.

\section{Funding Sources}

The authors have no funding to report.

\section{Author Contributions}

Z.H.F. conceived the study, participated in the design, screened literatures, assessed quality, collected the data, and drafted the manuscript. C.L.X. participated in the design, screened literatures, assessed quality, collected the data, performed statistical analyses, and drafted the manuscript. X.L. conceived the study, participated in the design, and helped draft the manuscript. L.B.G. and Z.Z.W. revised the paper. All authors read and approved the final manuscript (Z.Y.F. and C.L.X. were blind to each other in screening literatures and assessing quality).

\section{References}

1 Berkhemer OA, Fransen PS, Beumer D, van den Berg LA, Lingsma HF, Yoo AJ, et al. A randomized trial of intraarterial treatment for acute ischemic stroke. N Engl J Med. 2015;372:11-20.

2 Bracard S, Ducrocq X, Mas JL, Soudant M, Oppenheim C, Moulin T, et al. Mechanical thrombectomy after intravenous alteplase versus alteplase alone after stroke (THRA$\mathrm{CE})$ : a randomised controlled trial. Lancet Neurol. 2016;15:1138-47.

3 Campbell BC, Mitchell PJ, Kleinig TJ, Dewey HM, Churilov L, Yassi N, et al. Endovascular therapy for ischemic stroke with perfusion-imaging selection. N Engl J Med. 2015;372:1009-1018.

4 Goyal M, Demchuk AM, Menon BK, Eesa M, Rempel JL, Thornton T, et al. Randomized assessment of rapid endovascular treatment of ischemic stroke. N Engl J Med. 2015;49:1019-30.

5 Jovin TG, Chamorro A, Cobo E, de Miquel MA, Molina CA, Rovira A, et al. Thrombectomy within 8 hours after symptom onset in ischemic stroke. N Engl J Med. 2015;372: 2296-306.

6 Saver JL, Goyal M, Bonafe A, Diener HC, Levy EL, Pereira VM, et al. Stent-retriever thrombectomy after intravenous t-PA vs. t-PA alone in stroke. N Engl J Med. 2015; 372:2285-95.

7 Powers WJ, Derdeyn CP, Biller J, Coffey CS, Hoh BL, Jauch EC, et al. 2015 American Heart Association/American Stroke Association focused update of the 2013 guidelines for the early management of patients with acute ischemic stroke regarding endovascular treatment: a guideline for healthcare professionals from the American Heart Association/American Stroke Association. Stroke. 2015;46:3020-35.

8 Rubiera M, Alvarezsabín J, Ribo M, Montaner J, Santamarina E, Arenillas JF, et al. Predictors of early arterial reocclusion after tissue plasminogen activator-induced recanalization in acute ischemic stroke. Stroke. 2005;36:1452-6.

9 Yang M, Huo X, Miao Z, Wang Y. Platelet glycoprotein IIb/IIIa receptor inhibitor tirofiban in acute ischemic stroke. Drugs. 2019; 79(5):515-529.
10 Gruber P, Hlavica M, Berberat J, Victor Ineichen B, Diepers M, Nedeltchev K, et al. Acute administration of tirofiban versus aspirin in emergent carotid artery stenting. Interv Neuroradiol. 2018.25(2):219-24.

11 Huang S, Li W, et al. Preliminary results on safety and efficacy of intravenous tirofiban before endovascular thrombectomy for patients with acute ischemic stroke caused by large artery artherosclerosis. Eur Stroke J. 2018; 3(1): 103 . https://doi.org/10.1177/ 2396987318770127.

12 Huo X, Ming Y, Gao Feng, Wang A, Zhang Xiao, et al. Rescue tirofiban during mechanical thrombectomy for acute ischemic stroke patients with bridging intravenous thrombolysis. Stroke. 2019;50(1). https://doi. org/10.1161/str.50.suppl_1.TP51.

13 Kellert L, Hametner C, Rohde S, Bendszus M, Hacke W, Ringleb P, et al. Endovascular stroke therapy: tirofiban is associated with risk of fatal intracerebral hemorrhage and poor outcome. Stroke. 2013;44(5):1453-5.

14 Lee JI, Gliem M, Gerdes G, Turowski B, Kaschner M, Kraus B, et al. Safety of bridging antiplatelet therapy with the gpIIb-IIIa inhibitor tirofiban after emergency stenting in stroke. PLoS One. 2017;12(12): e0190218.

15 Luo Y, Yang Y, Xie Y, Yuan Z, Li X, Li J. Therapeutic effect of pre-operative tirofiban on patients with acute ischemic stroke with mechanical thrombectomy within 6-24 hours. Interv Neuroradiol. 2019;25(6):7059.

16 Pan X, Zheng D, Zheng Y, Chan PWL, Lin $\mathrm{Y}$, Zou J, et al. Safety and efficacy of tirofiban combined with endovascular treatment in acute ischemic stroke. Eur J Neurol. 2019; 26(8):1105-10.

17 Wu Y, Yin C, Yang J, Jiang L, Parsons MW, Lin L, et al. Endovascular thrombectomy: tirofiban increases bleeding risk in acute stroke patients. Stroke. 2018;49(11):27832785.

18 Yu T, Lin Y, Jin A, Zhang P, Zhou X, Fang $M$, et al. Safety and efficiency of low dose intra-arterial tirofiban in mechanical thrombectomy during acute ischemic stroke. Curr Neurovasc Res. 2018;15(2): 145-50.
19 Zhang S, Hao Y, Tian X, Zi W, Wang H, Yang D, et al. Safety of intra-arterial tirofiban administration in ischemic stroke patients after unsuccessful mechanical thrombectomy. J Vasc Interv Radiol. 2019;30:1417.e1.

20 Zhao W, Che R, Shang S, Wu C, Li C, Wu L, et al. Low-dose tirofiban improves functional outcome in acute ischemic stroke patients treated with endovascular thrombectomy. Stroke. 2017;48(12):3289-94.

21 Stang A. Critical evaluation of the Newcastle-Ottawa scale for the assessment of the quality of nonrandomized studies in metaanalyses. Eur J Epidemiol. 2010;25(9):6035.

22 Hacke W, Kaste M, Bluhmki E, Brozman M, Dávalos A, Guidetti D, et al. Thrombolysis with alteplase 3 to 4.5 hours after acute ischemic stroke. N Engl J Med. 2008;359(13): 1317-29.

23 Zaidat OO, Yoo AJ, Khatri P, Tomsick TA, von Kummer R, Saver JL, et al. Recommendations on angiographic revascularization grading standards for acute ischemic stroke: a consensus statement. Stroke. 2013;44: 2650-63.

24 van Swieten JC, Koudstaal PJ, Visser MC, Schouten HJ, van Gijn J. Interobserver agreement for the assessment of handicap in stroke patients. Stroke. 1988;19:604-7.

25 Higgins JPT, Green S. Cochrane handbook for systematic review of interventions (version 5.01). Wiley: The Cochrane Collaboration; 2008.

26 Siebler M, Hennerici MG, Schneider D, von Reutern GM, Seitz RJ, Röther J, et al. Safety of tirofiban in acute ischemic stroke: the SaTIS trial. Stroke. 2011;42:2388-92.

27 Torgano G, Zecca B, Monzani V, Maestroni A, Rossi P, Cazzaniga M, et al. Effect of intravenous tirofiban and aspirin in reducing short-term and long-term neurologic deficit in patients with ischemic stroke: a doubleblind randomized trial. Cerebrovasc Dis. 2010;29:275-81.

28 Fabris E, Kilic S, Schellings DAAM, Ten Berg JM, Kennedy MW, van Houwelingen $\mathrm{KG}$, et al. Long-term mortality and prehospital tirofiban treatment in patients with ST elevation myocardial infarction. Heart. 2017;103:1515-20. 
29 Saver JL, Jahan R, Levy EI, Jovin TG, Baxter B, Nogueira RG, et al. Solitaire flow restoration device versus the Merci Retriever in patients with acute ischaemic stroke (SWIFT): a randomised, parallel-group, non-inferiority trial. Lancet. 2012;380:1241-9.

30 Mangiafco S, Cellerini M, Nencini P, Gensini $\mathrm{G}$, Inzitari D. Intravenous tirofiban with intra-arterial urokinase and mechanical thrombolysis in stroke: preliminary experience in 11 cases. Stroke. 2005;36:2154-8.

31 Ihn YK, Sung JH, Kim BS. Intravenous glycoprotein IIb/IIIa inhibitor (tirofiban) fol- lowed by low-dose intra-arterial urokinase and mechanical thrombolysis for the treatment of acute stroke. Neuroradiol J. 2011; 24:907-13.

32 Seitz RJ, Hamzavi M, Junghans U, Ringleb PA, Schranz C, Siebler M. Thrombolysis with recombinant tissue plasminogen activator and tirofiban in stroke: preliminary observations. Stroke. 2003;34:1932-5.

33 Qureshi AI, Hussein HM, Abdelmoula M, Georgiadis AL, Janjua N. Subacute recanalization and reocclusion in patients with acute ischemic stroke following endovascu- lar treatment. Neurocrit Care. 2009;10:195203.

34 Choudhri TF, Hoh BL, Zerwes HG, Prestigiacomo CJ, Kim SC, Connolly ES Jr, et al. Reduced microvascular thrombosis and improved outcome in acute murine stroke by inhibiting GP IIb/IIIa receptor-mediated platelet aggregation. J Clin Invest. 1998;102: 1301-10.

35 Philipps J, Thomalla G, Glahn J, Schwarze M, Rother J. Treatment of progressive stroke with tirofiban-experience in 35 patients. Cerebrovasc Dis. 2009;28:435-8. 